Western University Scholarship@Western

Medical Biophysics Publications

Medical Biophysics Department

2-23-2015

\title{
Dynamic perfusion CT in brain tumors.
}

Timothy Pok Chi Yeung

Glenn Bauman

Slav Yartsev

Enrico Fainardi

David Macdonald

See next page for additional authors

Follow this and additional works at: https://ir.lib.uwo.ca/biophysicspub

Part of the Medical Biophysics Commons

Citation of this paper:

Yeung, Timothy Pok Chi; Bauman, Glenn; Yartsev, Slav; Fainardi, Enrico; Macdonald, David; and Lee, Ting-Yim, "Dynamic perfusion CT in brain tumors." (2015). Medical Biophysics Publications. 53.

https://ir.lib.uwo.ca/biophysicspub/53 
Authors

Timothy Pok Chi Yeung, Glenn Bauman, Slav Yartsev, Enrico Fainardi, David Macdonald, and Ting-Yim Lee 


\title{
Dynamic perfusion CT in brain tumors
}

\author{
Timothy Pok Chi Yeung a,b,c , Glenn Bauman a,b,d , Slav Yartseva,b,d , Enrico Fainardi ${ }^{\mathrm{e}}$, \\ David Macdonald ${ }^{\mathrm{b}, \mathrm{d}, \mathrm{f}}$, Ting-Yim Lee $\mathrm{a}^{\mathrm{a}, \mathrm{c}, \mathrm{d}, \mathrm{g}, \mathrm{h}, *}$
}

\footnotetext{
a Department of Medical Biophysics, The University of Western Ontario, 1151 Richmond Street N., London, Ontario, Canada N6A 5C1

${ }^{\mathrm{b}}$ London Regional Cancer Program, London Health Sciences Centre, 790 Commissioners Road East, London, ON, Canada N6A 5W9

${ }^{\mathrm{c}}$ Robarts Research Institute, The University of Western Ontario, 1151 Richmond St. N., London, Ontario, Canada N6A 5B7

d Department of Oncology, The University of Western Ontario, Victoria Hospital, London Health Sciences Centre, 790 Commissioners Road East, London, Ontario, Canada N6A 4 L6

e Azienda Ospedaliero-Universitaria di Ferrara, Neuroradiology Unit-Department of Neuroscience and Rehabilitation-Azienda, Ospedaliero-Universitaria di Ferrara-Arcispedale Sant'Anna, Via Aldo Moro, 8 - (Italy), Cona, Ferrara, Ferrara 44124 Italy

${ }_{\mathrm{f}}^{\mathrm{f}}$ Department of Clinical Neurological Sciences, The University of Western Ontario, University, Hospital, London Health Sciences Centre, 339 Windermere Road, London Ontario Canada N6A 5A5

${ }^{g}$ Department of Medical Imaging, The University of Western Ontario, Victoria Hospital, London Health Sciences Centre, London, Ontario, Canada N6A 5W9
}

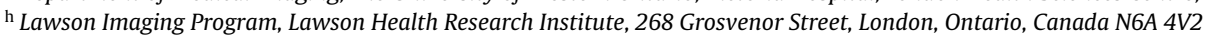

\section{A R T I C L E I N F O}

\section{Article history:}

Received 26 December 2014

Accepted 15 February 2015

\section{Keywords:}

Dynamic perfusion CT

Brain tumor

Blood flow

Blood volume

Permeability-surface area product

Adiabatic Approximation of Johnson and

Wilson Model

\begin{abstract}
A B S T R A C T
Dynamic perfusion CT (PCT) is an imaging technique for assessing the vascular supply and hemodynamics of brain tumors by measuring blood flow, blood volume, and permeability-surface area product. These PCT parameters provide information complementary to histopathologic assessments and have been used for grading brain tumors, distinguishing high-grade gliomas from other brain lesions, differentiating true progression from post-treatment effects, and predicting prognosis after treatments. In this review, the basic principles of PCT are described, and applications of PCT of brain tumors are discussed. The advantages and current challenges, along with possible solutions, of PCT are presented.
\end{abstract}

(c) 2015 Published by Elsevier Ireland Ltd.

\section{Introduction}

High-grade gliomas (HGGs), the most common primary brain tumors, are associated with high mortality rates despite aggressive treatments. The median survival for patients with glioblastomas, the most aggressive form of gliomas, is only 12-15 months [1]. HGGs are highly vascular tumors, and tumor vascularity is a determining pathologic hallmark of malignancy [2] that results in characteristic tumor contrast uptake on contrast-enhanced computed tomography (CT) or magnetic resonance imaging (MRI).

Perfusion imaging provides quantitative information about normal brain and tumor physiology that cannot be obtained from conventional morphological imaging. Dynamic perfusion CT (PCT)

\footnotetext{
* Corresponding author at: Department of Medical Biophysics, The University of Western Ontario, 1151 Richmond Street N., London, Ontario, Canada, N6A 5B7.

E-mail addresses: timothy.yeung@lhsc.on.ca (T.P.C. Yeung),

glenn.bauman@lhsc.on.ca (G. Bauman), slav.yartsev@lhsc.on.ca (S. Yartsev), henryfai@tin.it (E. Fainardi), david.macdonald@lhsc.on.ca (D. Macdonald), tlee@imaging.robarts.ca, tlee@lawsonimaging.ca (T.-Y. Lee).
}

is an imaging technique that can quantitatively assesses the vascular supply and permeability of brain tumors by measuring tumor blood flow (BF), blood volume (BV), and permeability-surface area product (PS). PCT is well-suited to study brain tumors due to its widespread availability and low cost, and it is relatively easy to implement compared to Positron emission tomography (PET) and MR perfusion. PCT has been used for grading gliomas, distinguishing gliomas from other brain lesions, differentiating tumor progression from treatment-induced effects, and predicting prognosis after treatments. This review describes the basic principles of PCT and its applications in neuro-oncology. The advantages and challenges of PCT as well as their solutions in brain tumor imaging are presented.

\section{Basic principles of PCT}

PCT acquires repeated images to track a bolus of iodinated contrast agent as it washes into and out of tissue via blood vessels. The efficient attenuation of X-rays by iodine increases CT image intensity, which is linearly proportional to the iodine concentration [3]. 
Table 1

A typical brain tumor PCT protocol.

\begin{tabular}{ll}
\hline Parameters & Specifications \\
\hline Contrast agent & $\bullet$ Iopamidol \\
Molecular weight of contrast agent & $\bullet 777 \mathrm{~g} / \mathrm{mol}(0.777 \mathrm{kDa})$ \\
Contrast concentration & $\bullet 300 \mathrm{mg}$ Iodine $/ \mathrm{ml}$ \\
Contrast dose & $\bullet 0.8 \mathrm{ml} / \mathrm{kg}(60-80 \mathrm{ml}$ in volume for a \\
& typical patient $)$ \\
Rate of contrast injection & $\bullet 2-4 \mathrm{ml} / \mathrm{s}$ \\
Scan duration & $\bullet 2-3 \mathrm{~min}$ \\
Temporal resolution & $\bullet$ First phase: $1-2 \mathrm{~s} /$ image for $45 \mathrm{~s}$ \\
& Second phase: $15 \mathrm{~s} /$ image for $105 \mathrm{~s}$ \\
Tube current & $\bullet 100-190 \mathrm{~mA}$ \\
Tube voltage & $\bullet 80 \mathrm{kV}$ \\
Scan coverage & $\bullet 4,8$, or $16 \mathrm{~cm}$ coverage with \\
& multi-detector CT \\
& $\bullet$ Shuttle mode can achieve whole \\
& brain coverage with 4 or $8 \mathrm{~cm}$ \\
Scan start time & multi-detector CT \\
\hline
\end{tabular}

The basis of PCT is to track the delivery of iodine by measuring changes in image intensity after the arrival of contrast bolus.

Table 1 illustrates a typical brain tumor PCT protocol. It is a twophase scan that takes two to three minutes. The first phase typically takes $45 \mathrm{~s}$ to capture the first pass of contrast and requires rapid acquisition of CT images (1-2 s/image) for accurate calculations of $\mathrm{BF}$ and BV. The second phase is required for the calculation of PS; it involves less frequent acquisition of CT images (10-15 s/image) to reduce imaging dose.

The calculation of PCT parameters consists of four steps:

1. Subtract baseline signal intensity (prior to contrast arrival) from each CT image to obtain tissue time-enhancement curves $C_{t}(t)$.

2. Select an input artery that supplies the brain to obtain the arterial input function $C_{a}(t)$.

3. Select a vein to correct partial volume averaging of the arterial input function $C_{a}(t)$ [4].

4. Calculate PCT parameters based on the chosen tracer kinetic model of the software.

The different tracer kinetics models can be categorized as follows:

1. Model-independent method based on indicator dilution theory that calculates BF and BV [5]. This is commonly used in dynamic susceptibility-contrast MR (DSC-MR). For brain tumor, the BV obtained has to be corrected for contrast leakage as described by Boxerman [6].

2. Compartmental model accounts for the rate of diffusional exchange of contrast between the intravascular space and the interstitial space (e.g. $K^{\text {trans }}$ ) besides BV [7]. This is commonly used in dynamic contrast-enhanced MR (DCE-MR). $K^{\text {trans }}$ is the product of $\mathrm{BF}$ and extraction efficiency $(E)$ and when $\mathrm{PS}$ « BF, as would be the case in brain tumor, it is equal to PS [8].

3. Distributed parameter model, which is commonly used in PCT, calculates BF, BV, and rate of diffusional exchange (PS or $K^{\text {trans }}$ ) between blood and tissue [8]. $K^{\text {trans }}$ can then be calculated as BF. $(1-\exp (-\mathrm{PS} / \mathrm{BF}))[8]$.

Here we describe the calculation of PCT parameters based on the distributed parameter model first proposed by Johnson and Wilson and subsequently simplified by St Lawrence and Lee with their adiabatic approximation [9]. The tissue-enhancement curve $C_{t}(t)$ can be expressed as:

$C_{t}(t)=B F \cdot C_{a}(t) \otimes R(t)$ where $\otimes$ is a convolution operator, and $R(t)$ represents the impulse residue function (IRF). Fig. 1 illustrates Eq. (1). The IRF describes the fraction of contrast that remains in the tissue as time progresses, following the injection of unit mass of contrast into the arterial input. The product of the IRF and BF is called the BF-scaled IRF, and it is solved by deconvolving the arterial input function $C_{a}(t)$ with the tissue enhancement curve $C_{t}(t)$. The BF-scaled impulse residue function has two distinct phases (Fig. 1). The first phase describes the retention of contrast in the tissue prior to any venous outflow; it has a peak height of $\mathrm{BF}(\mathrm{ml} / \mathrm{min} / 100 \mathrm{~g})$, a width that equals to the mean transit time (MTT, s), and an area that equals to $\mathrm{BV}(\mathrm{ml} / 100 \mathrm{~g})$. The second phase describes the backflux of contrast from the interstitial space into the bloodstream. It starts at a height of the extraction fraction $(E)$ and decays monoexponentially with time. PS can be calculated as PS $=-\mathrm{BF} \cdot \ln (1-E)$. PS is the unidirectional diffusional flux of contrast from the intravascular space into the interstitial space across all "openings" of the permeable capillary endothelium.

\section{Clinical applications}

\subsection{Correlation with histopathologic markers and tumor grade}

Angiogenesis is important for histopathologic grading of gliomas by providing valuable information for treatment selections and prediction of treatment response. Histopathologic grading of gliomas using biopsied specimens can be prone to sampling error due to the heterogeneity of tumors. It has been shown that $60 \%$ of anaplastic astrocytomas (grade III) were upgraded to glioblastoma (grade IV) after comparing the biopsied specimens with the resected tumors [10].

Microvascular density (MVD) and microvascular cellular proliferation (MVCP) are histopathologic markers of angiogenesis. PCT measures of $\mathrm{BF}$ and $\mathrm{BV}$ have shown significant correlations with $\operatorname{MVD}(r=0.527$ and 0.649 , respectively; $P<0.02)$, while PS showed a significant correlation with $\operatorname{MVCP}(r=0.647, P=0.001)$ [11]. These results suggest that regions of higher angiogenic activities (i.e. more aggressive) could be localized by maps of BF, BV, and PS to serve to guide biopsy or resection. While statistically significant, PCT correlations with MVD and MVCP were moderate, possibly because MVD and MVCP cannot fully reflect the complexity of tumor vessels, which are tortuous and variable in size. MR perfusion measure of relative $\mathrm{BV}(\mathrm{rBV})$ showed higher correlation with microvessel area (MVA) than MVD ( $r=0.83$ and 0.32 , respectively; $P \leq 0.05)$ [12]. More importantly, MVA and rBV correlated with overall survival $(\mathrm{OS})(P<0.02)$ and MVD did not $(P=0.17)$, suggesting rBV and MVA could be superior to MVD for predicting OS. MVA can better reflect the tortuosity and size of vessels, but MVA is not measured routinely because it is time-consuming and labor-intensive. To date, correlations between MVA and PCT have not yet been reported.

Jain et al. explored the relationships between different PCT parameters and the expression levels of genes associated with angiogenesis in glioblastomas [13]. BV and/or PS correlated positively with some proangiogenic genes (e.g. VEGFR-2) and negatively with some anti-angiogenic genes (e.g. VASH2 and C3), suggesting a molecular genetic basis for using PCT to assess glioblastomas.

A number of studies have used PCT for tumor grading. In general, PCT of HGGs (grade III and IV) demonstrated higher BF, BV, and PS than low-grade gliomas (grade II) [11,14-22]. Figs. 2 and 3 illustrate MR and PCT studies of patients with low-grade glioma and HGG respectively. Table 2 shows the reported sensitivities and specificities of using PCT to differentiate HGGs versus low-grade gliomas. PCT has also been reported to differentiate grade III from grade IV gliomas with PS demonstrating better predictability than BV in 

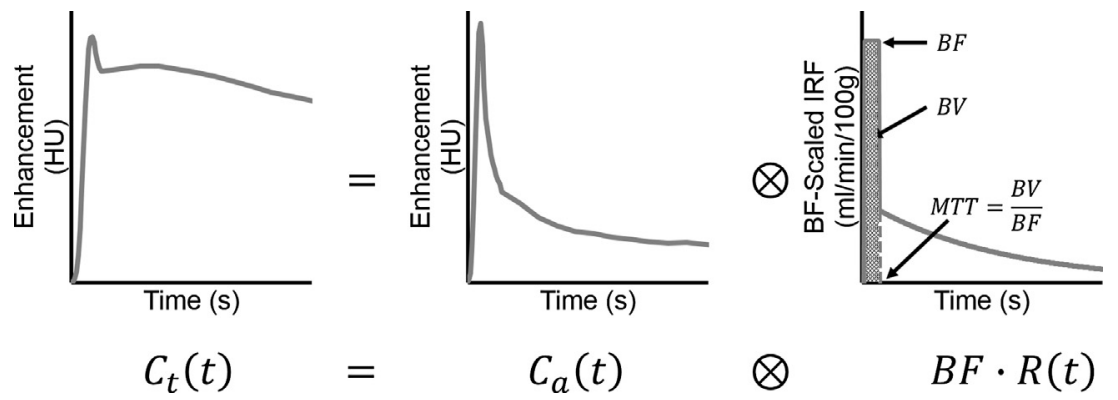

Fig. 1. Graphical illustration showing how different CT perfusion parameters are calculated from a tissue enhancement curve $C_{t}(t)$ and arterial input function $C_{a}(t)$.

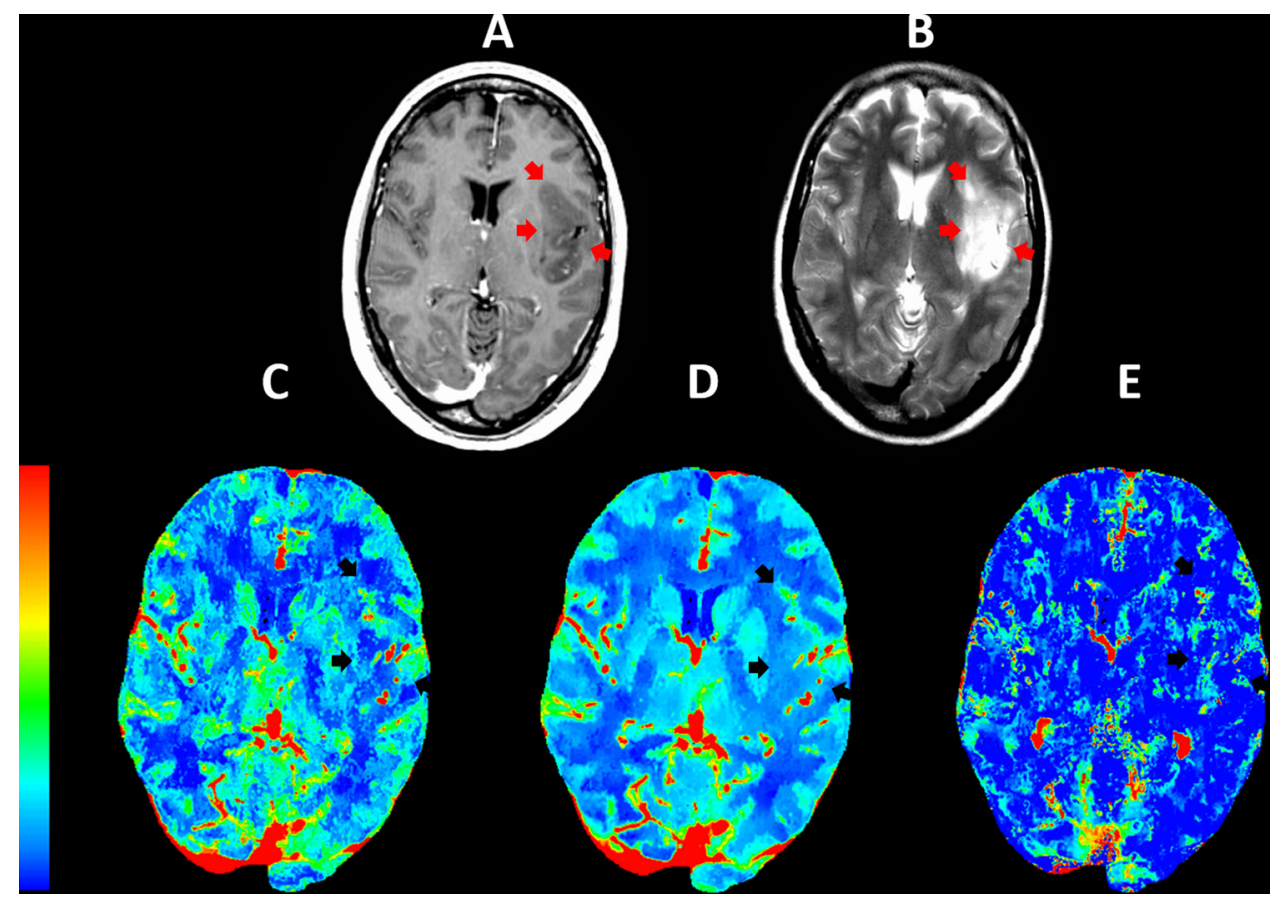

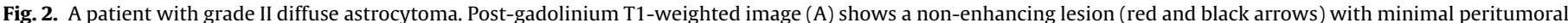

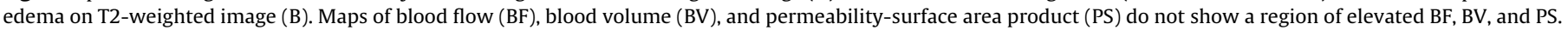

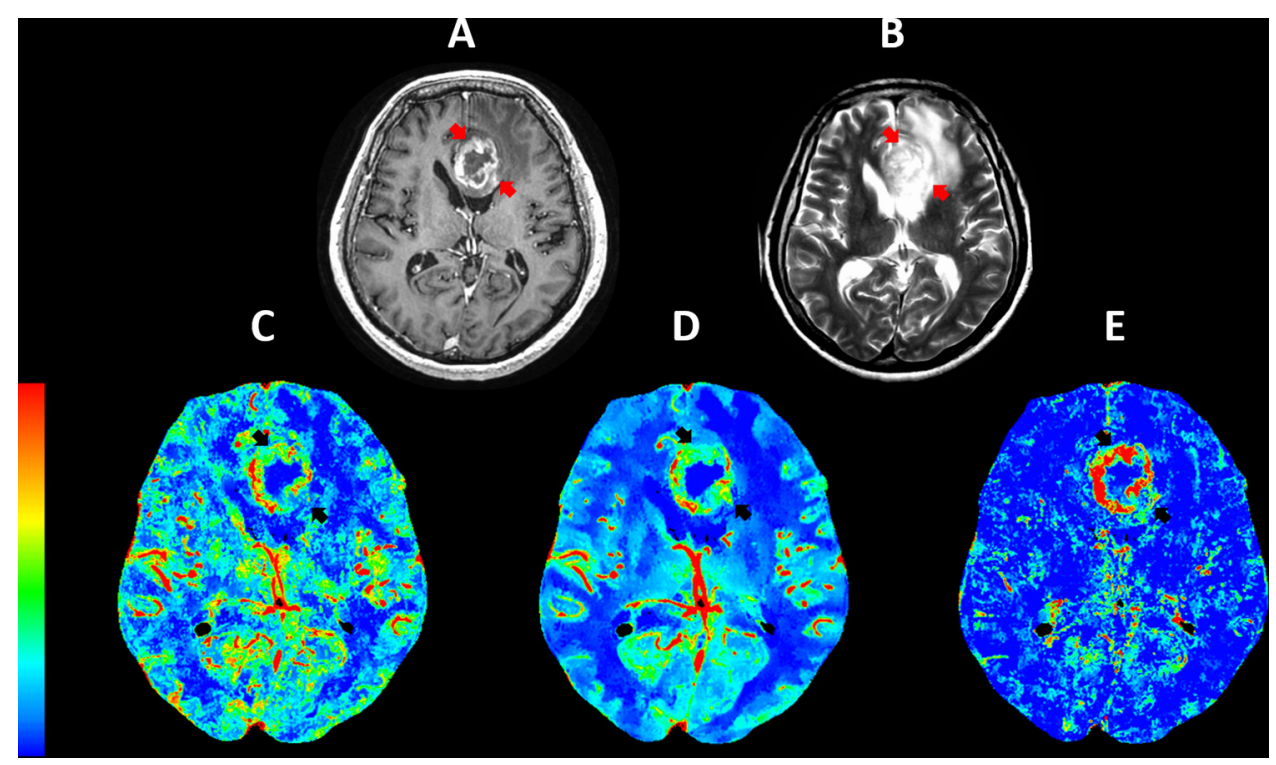

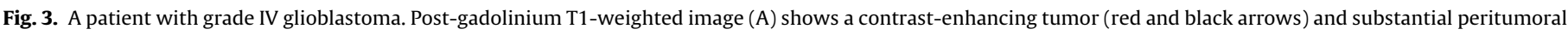
edema on T2-weighted image (B). Maps of blood flow (BF), blood volume (BV), and permeability-surface area product (PS) shows a rim with high BF, BV, and PS.

Please cite this article in press as: Yeung TPC, et al. Dynamic perfusion CT in brain tumors. Eur J Radiol (2015), http://dx.doi.org/10.1016/j.ejrad.2015.02.012 
Table 2

The performance of PCT in differentiating high vs. low grade gliomas.

\begin{tabular}{llcl}
\hline Parameters & Sensitivity ranges (\%) & Specificity ranges (\%) & References \\
\hline $\mathrm{BF}^{\mathrm{a}}$ & $71-91$ & $82-100$ & {$[14,15,18,22]$} \\
$\mathrm{BV}^{\mathrm{a}}$ & $83-100$ & $75-100$ & {$[14,15,18,19,22]$} \\
$\mathrm{PS}$ & 83 & 100 & {$[19]$} \\
MTT & 93 & 40 & {$[18]$} \\
$K^{\text {trans }}$ & 97 & 100 & {$[14,22]$}
\end{tabular}

Abbreviations: BF, blood flow; BV, blood volume; PS, permeability-surface area product; MTT, mean transit time; $K^{\text {trans }}$, transfer constant.

a BF and BV refer to absolute values or absolute tumor BF and BV normalized to $\mathrm{BF}$ and $\mathrm{BV}$ in the normal appearing white matter or contralateral brain region.

this regard ( $C$-statistic $=0.926$ and 0.787 , respectively) [17]. This is consistent with the correlation between PS and MVCP [11] in that neovascularization is a criterion for diagnosing grade IV gliomas, but not grade III gliomas, suggesting PS is sensitive to detecting immature and leaky tumor vessels. In addition to grading, PS has been shown to improve interobserver agreement of tumor boundary delineation, which is particularly important for tumor targeting by radiotherapy [23].

Up to $9 \%$ of HGGs are non-enhancing on post-gadolinium T1weighted MR, which can be mistaken as low-grade gliomas [24]. Beppu et al. showed that PCT measurement of BV could differentiate non-enhancing grade III gliomas from grade II gliomas with a sensitivity and specificity of $90.9 \%$ and $83.3 \%$, respectively [15]. All these studies support the use of PCT as a noninvasive surrogate of tumor grade, which can potentially be used to guide biopsy and resection of gliomas and potentially avoid under-grading nonenhancing HGGs.

\subsection{Differentiation of high-grade gliomas from other brain lesions}

There are non-malignant and malignant lesions that can appear similar to HGGs on contrast-enhanced MR images. Tumefactive demyelinating lesions (TDLs) are solitary lesions greater than $2 \mathrm{~cm}$ that mimic HGGs on contrast-enhanced MR images [25], and can also be confused with HGGs on histopathology [26]. Differentiation of TDLs from HGGs may avoid unnecessary biopsy and resection of viable brain tissue. Using PCT, TDLs showed significantly lower BF, $\mathrm{BV}$, and PS than HGGs [27]. These results are consistent with the observations that TDLs are characterized by normal or inflamed vessels and a lack of vascular proliferation while the latter is common in HGGs [26].

Primary brain lymphomas, brain metastases, and HGGs are malignant lesions that can appear similar to each other on MR images. HGGs showed significantly higher BV and BF than primary brain lymphomas, suggesting PCT may have utility in differentiating the two entities $[22,28]$. However, Fainardi et al. showed that there was no significant difference in tumor BV and PS between HGGs and metastases [16]. Previous DSC-MR studies showed that tumor BV values from brain metastases were either similar to or lower than HGGs $[29,30]$. Thus, it may be difficult to distinguish metastases from HGGs using tumor BV. DSC-MR studies that focused on the peritumoral edema regions of tumors (hyperintense regions on T2-weighted MR) found that BV values in these regions were significantly higher in HGGs than brain metastases [30,31]. This observation suggests a possibility to distinguish HGG and brain metastases by evaluating BV in the peritumoral edema region. The high $\mathrm{BV}$ in the peritumoral edema region of HGG could be attributed to its infiltrative growth while tumor infiltration is not a characteristic feature of brain metastasis [32].
Table 3

Differentiation of treatment-induced necrosis from true progression of brain tumors.

\begin{tabular}{llll}
\hline Parameters & Sensitivity ranges (\%) & Specificity ranges (\%) & References \\
\hline${ }^{a}$ Relative BF & $94 \%$ & $88 \%$ & {$[38]$} \\
a Relative BV & $71-83 \%$ & $90-100 \%$ & {$[38-40]$} \\
PS & $82 \%$ & $82 \%$ & {$[39]$} \\
Relative MTT & $94 \%$ & $75 \%$ & {$[38]$}
\end{tabular}

Abbreviations: BF, blood flow; BV, blood volume; PS, permeability-surface area product; MTT, mean transit time.

a Relative BF and BV refer to absolute tumor BF and BV normalized to BF and BV in the normal appearing white matter or contralateral brain region.

\subsection{Differentiation of true progression from post-treatment effects}

An increase in the size of the contrast-enhancing tumor is a radiologic measure of progression, but the effects of radiotherapy can also mimic the appearance of true progression on contrast-enhanced MR images [33]. Pseudoprogression and treatment-induced necrosis (TIN) are two post-radiotherapy scenarios that mimic true progression on MR. It is imperative to differentiate these entities accurately since they are managed differently.

Although the mechanism of pseudoprogression is unclear, it is believed to be induced by a local inflammatory reaction, edema, and increased vessel permeability that lead to contrast enhancement on imaging [33]. Previous DSC-MR studies showed that true progression had significantly higher relative BV (rBV) than pseudoprogression [34,35] and TIN [36,37]. Using PCT, true progression showed significantly higher values of $\mathrm{rBF}, \mathrm{rBV}$, and PS than TIN [38-40]. Sensitivities and specificities of $>80 \%$ were measured using PCT to differentiate true progression from TIN in brain tumors (Table 3). Fig. 4 shows a PCT study of a patient with progression 6 months after radiotherapy, and Fig. 5 shows a patient with histopathologically confirmed radiation necrosis 3 months after radiotherapy.

PCT offers an advantage over MR perfusion in this regard since it can measure all three vascular parameters-BF, BV and PS, which is technically challenging for DSC-MR and DCE-MR. True progressive brain tumors are malignancies with increased vascularity, immature leaky vessels, and upregulated VEGF expression resulting in higher BF, BV and PS. On the other hand, the moderate PS observed in TIN is likely mediated by the release of VEGF in the pathogenesis of radiation necrosis [41]. In addition, susceptibility artefacts from blood products (hemorrhage, thrombus) and calcification can potentially complicate the analysis of DSC-MR studies while PCT is not affected by these artefacts $[39,42]$.

\subsection{Prediction of prognosis}

Recently, PCT parameters have been used to predict overall survival (OS) after surgery/biopsy, radiotherapy, and temozolomide chemotherapy. Pre-treatment PCT exams showed that tumor relative BV alone [43], PS alone [44], and relative BV+PS were all predictive of OS $[43,44]$. More importantly, rBV alone and rBV + PS remained significant predictors of OS even after adjusting for classical prognostic factors (age, Karnofsky performance status, extent of resection, and grade) [43], suggesting that PCT parameters could improve the prediction of OS.

Local recurrence within $2 \mathrm{~cm}$ of the irradiated volume predominates post-radiotherapy, making imaging assessments of both the contrast-enhancing tumor and the peritumoral edema region critical [45]. Yeung et al. showed that patients with shorter OS had higher BV and PS in both regions than patients with longer OS (Fig. 6), and that post-radiotherapy BV in the peritumoral edema 


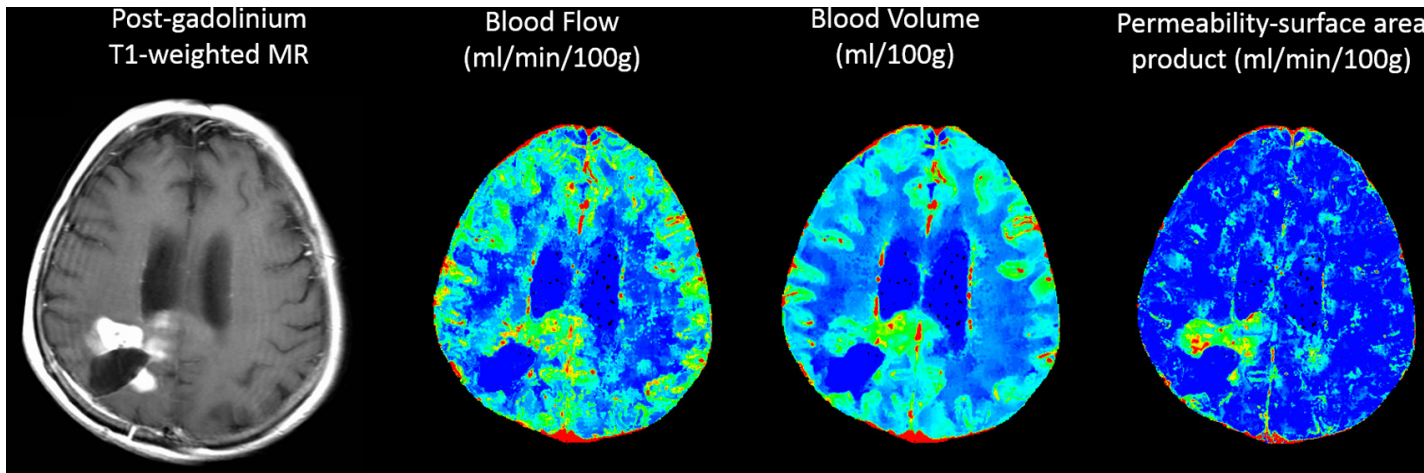

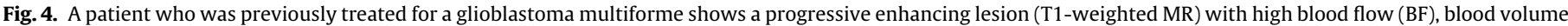
(BV), and permeability-surface area product (PS) suggesting progressive tumor around the surgical cavity at 6 months post-radiotherapy.

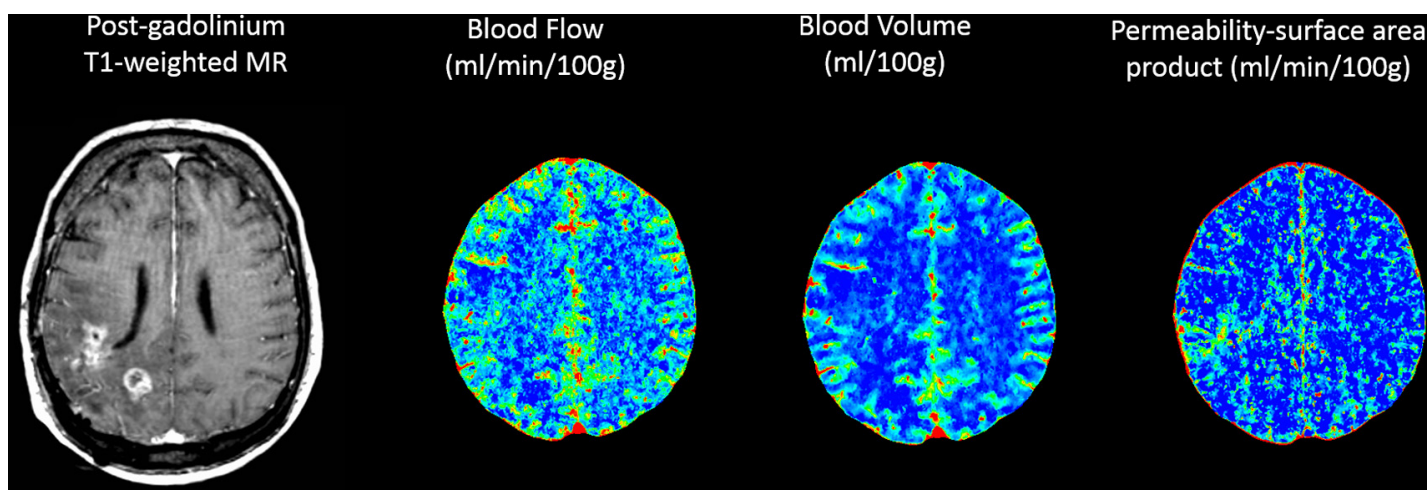

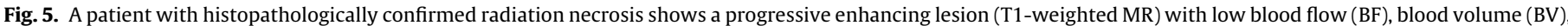
and permeability-surface area product (PS).

region could predict 24 months OS with sensitivities and specificities $>80 \%[46]$. Similar results have been demonstrated in DSC-MR studies $[47,48]$. These findings highlight the importance of paying attention to the peritumoral edema region. Radiotherapy targeting of the peritumoral edema region with PCT image-guidance may be a viable option that is worth exploring. PCT can play an important role in radiation oncology since CT imaging is required for radiotherapy treatment planning.

\section{Discussion: strengths and challenges of PCT}

DSC-MR is currently the most commonly used perfusion imaging technique for assessing brain tumors. However, PCT has several advantages that make it a useful alternative functional imaging tool. The linear relationship between signal intensity (HU) and iodine concentration is a major strength of PCT. DSC-MR with $\mathrm{T} 2$ or $\mathrm{T} 2{ }^{*}$-weighting is prone to two types of artefacts. DSC-MR

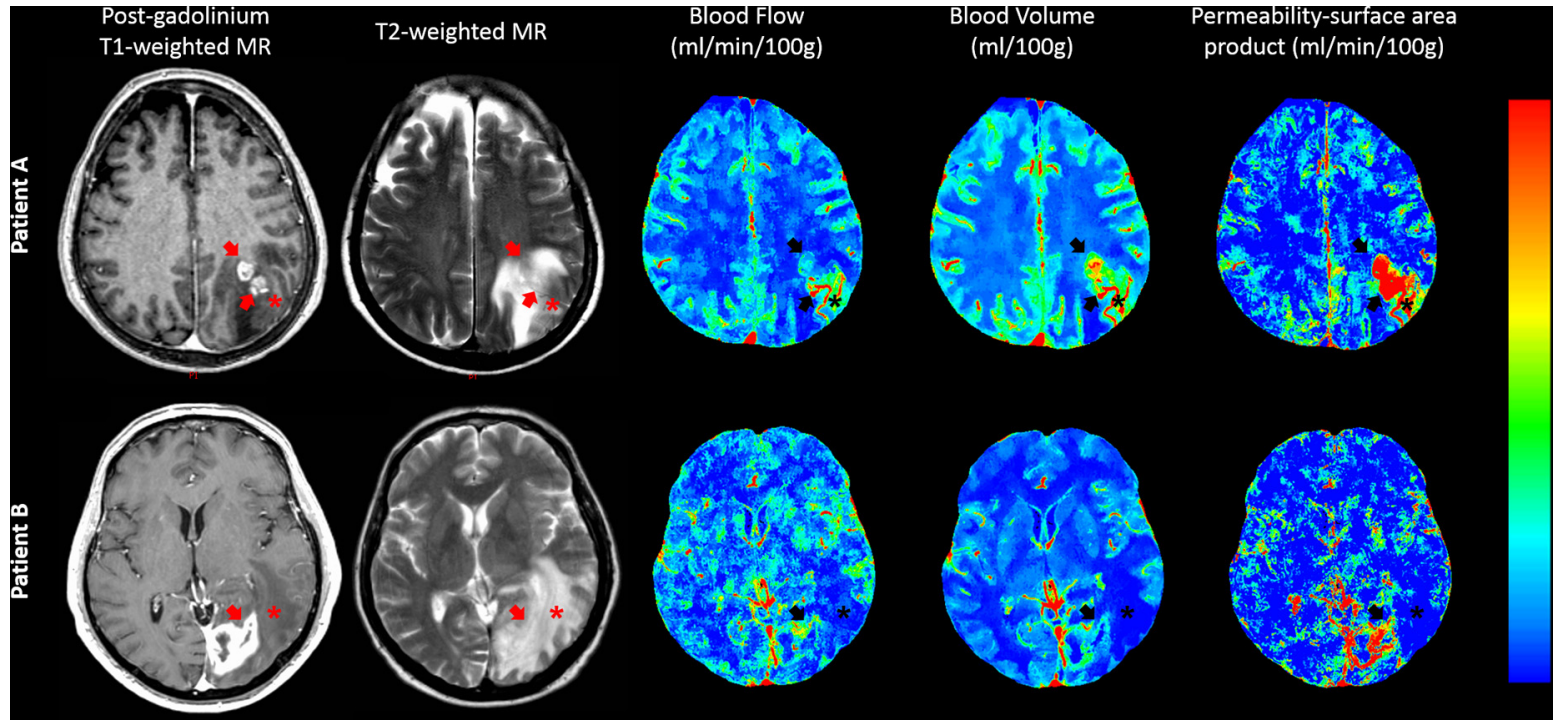

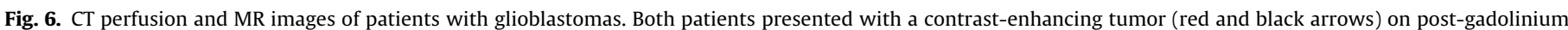

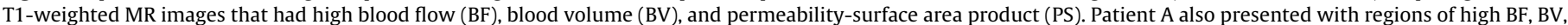

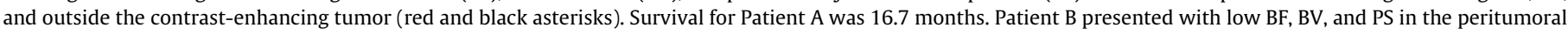
region outside the contrast-enhancing tumor (red and black asterisks). Survival for patient B was 41.6 months.

Please cite this article in press as: Yeung TPC, et al. Dynamic perfusion CT in brain tumors. Eur J Radiol (2015), http://dx.doi.org/10.1016/j.ejrad.2015.02.012 
measurement of $\mathrm{BV}$ depends on the compartmentalization of contrast within blood vessels. In brain tumors where considerable amount of contrast can leak into the interstitial space, the susceptibility-contrast signal intensity loss can be masked by the competing T1 effects [6]. DSC-MR measurement leads to an underestimation of BV if it is not addressed by (1) pre-saturating the brain parenchyma with a pre-loading dose of contrast, (2) dual-echo sequence, or (3) post-processing correction [6,42]. This effect also makes the quantification of tumor permeability technically challenging. In addition, the susceptibility artefacts created by blood products (from hemorrhage and thrombus) in tumors are other factors that can affect the quantification of BV. PCT is clearly advantageous in this respect.

The second major advantage of PCT is the capability to measure $\mathrm{BF}, \mathrm{BV}$, and PS from a single scan. The measurements of $\mathrm{BF}$ and $\mathrm{BV}$ require rapid image acquisitions (1-2 s per image), which is technically challenging for T1-weighted DCE-MR. For DSC-MR, the effect of contrast extravasation on signal intensity makes the calculation of PS difficult to achieve as discussed above. Furthermore, measuring the arterial input function needed for calculating absolute values of $\mathrm{BF}$ and $\mathrm{BV}$ is problematic with MR perfusion due to the trade-off between spatial and temporal resolutions. The high spatial and temporal resolutions of CT scanning and the linearity between iodine concentration and $\mathrm{CT}$ image intensity enable the calculation of BF, BV, and PS from a single PCT study.

MR is the standard of care for brain tumor patients; hence, the follow-up of patients with MR perfusion is more convenient than PCT as perfusion imaging can be included as part of a single multi-parametric MR study. The clinical use of PCT has also been slow to progress due to two major limitations. Radiation dose is of concern when performing a PCT scan. The effective doses with current techniques are between 2.5 and $5.5 \mathrm{mSv}$ for tube currents of 100-190 mA [49]. Recent advances in adaptive statistical iterative reconstruction can reduce imaging dose without compromising the diagnostic acceptability [50]. An image filtering technique called principal component analysis can also improve PCT image quality; thereby presenting another opportunity for further dose reduction [51]. The limited scan coverage ( $2-4 \mathrm{~cm}$ coverage) is another limitation of PCT, making whole tumor assessment difficult. However, the advent of shuttle mode imaging and large multi-row detector technology enables whole brain PCT coverage [52,53]. Differences in PCT protocol (e.g. PCT scan duration) and calculation software could lead to disagreements in the values of PCT parameters [49,54]. Therefore, standardization of PCT protocol and software are critical for cross-study comparison.

\section{Conclusions}

PCT can provide tumor hemodynamics information for preoperative tumor grading, response assessment, and OS prediction. Results from PCT studies are comparable to those performed with MR perfusion. PCT is technically less demanding than MR perfusion and provides measurements of BF, BV, and PS from a single scan. The obstacles of limited scan coverage and radiation dose are being addressed by recent advances in CT imaging. In our opinion, PCT has reached sufficient technical maturity for use as a routine functional imaging tool for brain tumor assessment.

\section{Conflict of interest}

T-Y Lee licenses CT Perfusion software to GE Healthcare and receives royalties from the license. The software was used to generate the Figures in the article. Other authors do not have conflicts to declare.

\section{References}

[1] Wen PY, Kesari S. Malignant gliomas in adults. N Engl J Med 2008;359:492-507.

[2] Jain RK, di Tomaso E, Duda DG, Loeffler JS, Sorensen a G, Batchelor TT. Angiogenesis in brain tumours. Nat Rev Neurosci 2007;8:610-22.

[3] Miles KA, Young H, Chica SL, Esser PD. Quantitative contrast-enhanced computed tomography: is there a need for system calibration? Eur Radiol 2007; $17: 919-26$.

[4] Cenic A, Nabavi DG, Craen RA, Gelb AW, Lee T-Y. Dynamic CT measurement of cerebral blood flow: a validation study. AJNR Am J Neuroradiol 1999;20:63-73.

[5] Østergaard L. Cerebral perfusion imaging by bolus tracking. Top Magn Reson Imaging 2004;15:3-9.

[6] Boxerman J, Schmainda KM, Weisskoff R. Relative cerebral blood volume maps corrected for contrast agent extravasation significantly correlate with glioma tumor grade, whereas uncorrected maps do not. AJNR Am J Neuroradiol 2006;27:859-67

[7] Tofts P, Brix G, Buckley DL, et al. Estimating kinetic parameters from dynamic contrast-enhanced T1-weighted MRI of a diffusable tracer: standardized quantities and symbols. J Magn Reson Imaging 1999;10:223-32.

[8] Lee T-Y, Purdie T, Stewart E. CT imaging of angiogenesis. Q J Nucl Med 2003;41:171-87.

[9] St Lawrence K, Lee T-Y. An adiabatic approximation to the tissue homogeneity model for water exchange in the brain: I. theoretical derivation. J Cereb Blood Flow Metab 1998;18:1365-77.

[10] Jackson RJ, Fuller GN, Abi-Said D, et al. Limitations of stereotactic biopsy in the initial management of gliomas. Neuro Oncol 2001;3:193-200

[11] Jain R, Gutierrez J, Narang J, et al. In vivo correlation of tumor blood volume and permeability with histologic and molecular angiogenic markers in gliomas. AJNR Am J Neuroradiol 2011;32:388-94.

[12] Hu LS, Eschbacher JM, Dueck a C, et al. Correlations between perfusion MR imaging cerebral blood volume, microvessel quantification, and clinical outcome using stereotactic analysis in recurrent high-grade glioma. AJNR Am J Neuroradiol 2012;33:69-76.

[13] Jain R, Poisson L, Narang J, et al. Correlation of perfusion parameters with genes related to angiogenesis regulation in glioblastoma: a feasibility study. AJNR Am J Neuroradiol 2012;33:1343-8.

[14] Xyda A, Haberland U, Klotz E, et al. Brain volume perfusion CT performed with 128-detector row CT system in patients with cerebral gliomas: a feasibility study. Eur Radiol 2011;21:1811-9.

[15] Beppu T, Sasaki M, Kudo K, et al. Prediction of malignancy grading using computed tomography perfusion imaging in nonenhancing supratentorial gliomas. J Neurooncol 2011;103:619-27.

[16] Fainardi E, Biase FDi, Borrelli M, et al. Potential role of CT perfusion parameters in the identification of solitary intra-axial brain tumor grading. Acta Neurochir (Wien) 2010;106:283-7.

[17] Jain R, Ellika SK, Scarpace L, et al. Quantitative estimation of permeability surface-area product in astroglial brain tumors using perfusion CT and correlation with histopathologic grade. AJNR Am J Neuroradiol 2008;29:694-700.

[18] Ellika SK, Jain R, Patel SC, et al. Role of perfusion CT in glioma grading and comparison with conventional MR imaging features. AJNR Am J Neuroradiol 2007;28:1981-7.

[19] Ding B, Ling HW, Chen KM, Jiang H, Zhu YB. Comparison of cerebral blood volume and permeability in preoperative grading of intracranial glioma using CT perfusion imaging. Neuroradiology 2006;48:773-81.

[20] Jain R, Narang J, Gutierrez J, et al. Correlation of immunohistologic and perfusion vascular parameters with MR contrast enhancement using imageguided biopsy specimens in gliomas. Acad Radiol 2011;18:955-62.

[21] Lee Y-J, Ahn K-J, Kim B-S, Yoo W-J. Role of perfusion CT in differentiating between various cerebral masses using normalized permeability surface area product and cerebral blood volume. Clin Imaging 2012;36:680-7.

[22] Xyda A, Haberland U, Klotz E, et al. Diagnostic performance of whole brain volume perfusion $\mathrm{CT}$ in intra-axial brain tumors: preoperative classification accuracy and histopathologic correlation. Eur J Radiol 2012;81:4105-11.

[23] Bisdas S, Yang X, Lim CCT, Vogl TJ, Koh TS. Delineation and segmentation of cerebral tumors by mapping blood-brain barrier disruption with dynamic contrast-enhanced CT and tracer kinetics modeling-a feasibility study. Eur Radiol 2008; 18:143-51.

[24] Scott J, Brasher P, Sevick R, Rewcastle N, Forsyth P. How often are nonenhancing supratentorial gliomas malignant? A population study. Neurology 2002;59:947-9.

[25] Given C, Stevens B, Lee C. The MRI appearance of tumefactive demyelinating lesions. AJR Am J Roentgenol 2004;182:195-9.

[26] Sugita Y, Terasaki M, Shigemori M, Sakata K, Morimatsu M. Acute focal demyelinating disease simulating brain tumors: histopathologic guidelines for an accurate diagnosis. Neuropathology 2001;21:25-31.

[27] Jain R, Ellika S, Lehman NL, et al. Can permeability measurements add to blood volume measurements in differentiating tumefactive demyelinating lesions from high grade gliomas using perfusion CT? J Neurooncol 2010;97: 383-8.

[28] Schramm P, Klotz E, Knauth M, Tronnier V, Hartmann M. Dynamic CT perfusion imaging of intra-axial brain tumours: differentiation of high-grade gliomas from primary CNS lymphomas. Eur Radiol 2010;20:2482-90.

[29] Young GS, Setayesh K. Spin-echo echo-planar perfusion MR imaging in the differential diagnosis of solitary enhancing brain lesions: distinguishing solitary metastases from primary glioma. AJNR Am J Neuroradiol 2009;30:575-7. 
[30] Mouthuy N, Cosnard G, Abarca-Quinones J, Michoux N. Multiparametric magnetic resonance imaging to differentiate high-grade gliomas and brain metastases. J Neuroradiol 2011;39:301-7.

[31] Lehmann P, Saliou G, de Marco G, et al. Cerebral peritumoral oedema study: does a single dynamic MR sequence assessing perfusion and permeability can help to differentiate glioblastoma from metastasis? Eur J Radiol 2012;81:522-7.

[32] Raore B, Schniederjan M, Prabhu R, Brat DJ, Shu H-K, Olson JJ. Metastasis infiltration: an investigation of the postoperative brain-tumor interface. Int J Radiat Oncol Biol Phys 2011;81:1075-80.

[33] Brandsma D, Stalpers L, Taal W, Sminia P, van den Bent MJ. Clinical features, mechanisms, and management of pseudoprogression in malignant gliomas. Lancet Oncol 2008;9:453-61.

[34] Kong D-S, Kim ST, Kim E-H, et al. Diagnostic dilemma of pseudoprogression in the treatment of newly diagnosed glioblastomas: the role of assessing relative cerebral blood flow volume and oxygen-6-methylguanine-DNA methyltransferase promoter methylation status. AJNR Am J Neuroradiol 2011;32: 382-7.

[35] Gahramanov S, Raslan AM, Muldoon LL, et al. Potential for differentiation of pseudoprogression from true tumor progression with dynamic susceptibilityweighted contrast-enhanced magnetic resonance imaging using ferumoxytol vs. gadoteridol: a pilot study. Int J Radiat Oncol Biol Phys 2011;79:514-23.

[36] Hu LS, Eschbacher JM, Heiserman JE, et al. Reevaluating the imaging definition of tumor progression: perfusion MRI quantifies recurrent glioblastoma tumor fraction, pseudoprogression, and radiation necrosis to predict survival. Neuro Oncol 2012;14:919-30.

[37] Hu LS, Baxter LC, Smith Ka, et al. Relative cerebral blood volume values to differentiate high-grade glioma recurrence from posttreatment radiation effect: direct correlation between image-guided tissue histopathology and localized dynamic susceptibility-weighted contrast-enhanced perfusion. AJNR Am J Neuroradiol 2009;30:552-8.

[38] Jain R, Scarpace L, Ellika S, et al. First-pass perfusion computed tomography: Initial experience in differentiating recurrent brain tumors from radiation effects and radiation necrosis. Neurosurgery 2007;61:778-87.

[39] Jain R, Narang J, Schultz L, et al. Permeability estimates in histopathologyproved treatment-induced necrosis using perfusion CT: can these add to other perfusion parameters in differentiating from recurrent/progressive tumors? AJNR Am J Neuroradiol 2011;32:658-63.

[40] Vidiri A, Guerrisi A, Pinzi V, et al. Perfusion Computed Tomography (PCT) adopting different perfusion metrics: recurrence of brain metastasis or radiation necrosis? Eur J Radiol 2012;81:1246-52.
[41] Levin VA, Bidaut L, Hou P, et al. Randomized double-blind placebo-controlled trial of bevacizumab therapy for radiation necrosis of the central nervous system. Int J Radiat Oncol Biol Phys 2011;79:1487-95.

[42] Jain R. Perfusion CT imaging of brain tumors: an overview. AJNR Am J Neuroradiol 2011;32:1570-7

[43] Jain R, Narang J, Griffith B, et al. Prognostic vascular imaging biomarkers in highgrade gliomas: tumor permeability as an adjunct to blood volume estimates. Acad Radiol 2013:20:478-85.

[44] Shankar JJS, Woulfe J, Silva V Da, Nguyen TB. Evaluation of perfusion CT in grading and prognostication of high-grade gliomas at diagnosis: a pilot study. AJR Am J Roentgenol 2013;200:W504-9.

[45] Wen PY, Macdonald DR, Reardon Da, et al. Updated response assessment criteria for high-grade gliomas: response assessment in neuro-oncology working group. J Clin Oncol 2010;28:1963-72.

[46] Yeung TPC, Wang Y, He W, et al. Survival prediction in high-grade gliomas using CT perfusion imaging. J Neurooncol 2015. Under Review.

[47] Mangla R, Singh G, Ziegelitz D, et al. Changes in relative cerebral blood volume 1 month after radiation-temozolomide therapy can help predict overall survival in patients with glioblastoma. Radiology 2010;256:575-84.

[48] Jain R, Poisson LM, Gutman D, et al. Outcome prediction in patients with glioblastoma by using imaging, clinical, and genomic biomarkers: focus on the nonenhancing component of the tumor. Radiology 2014;272:484-93.

[49] Yeung TPC, Yartsev S, Bauman G, He W, Fainardi E, Lee T-Y. The effect of scan duration on the measurement of perfusion parameters in CT perfusion studies of brain tumors. Acad Radiol 2013:20:59-65.

[50] Kilic K, Erbas G, Guryildirim M, Arac M, Ilgit E, Coskun B. Lowering the dose in head CT using adaptive statistical iterative reconstruction. AJNR Am J Neuroradiol 2011;32:1578-82.

[51] Yeung TPC, Dekaban M, De Haan N, et al. Improving quantitative CT perfusion parameter measurements using principal component analysis. Acad Radiol 2014;21:624-32.

[52] Chen T, Guo D, Fang Z, Zhong W, Zhao J, Jiang Y. Preliminary study of wholebrain $\mathrm{CT}$ perfusion imaging in patients with intracranial tumours adjacent to large blood vessels. Clin Radiol 2014;69:e25-32.

[53] Youn SW, Kim JH, Weon Y-C, Kim SH, Han M-K, Bae H-J. Perfusion CT of the brain using 40-mm-wide detector and toggling table technique for initial imaging of acute stroke. AJR Am J Roentgenol 2008;191:W120-6.

[54] Goh V, Halligan S, Bartram CI. Quantitative tumor perfusion assessment with multidetector CT: are measurements from two commercial software packages interchangeable? Radiology 2007;242:777-82. 